\title{
A lesson on paper
}

\author{
This year's International Physics Olympiad - ably assisted by over 1,000 paper transistors - could help inspire the \\ next generation of scientists, engineers and technicians.
}

R ecent developments in artificial intelligence, big data and the Internet of Things - combined with a growing awareness of the ways such technology could benefit society - have put computer science education firmly in the spotlight. Back in November, for example, a report from the Royal Society in the UK called for a tenfold increase in funding for the subject in schools ${ }^{1}$. And a few months earlier, during a roundtable discussion at the White House, Tim Cook, the chief executive of Apple, told President Trump that "coding should be a requirement in every public school" . There are, though, two sides to this technology the software and the hardware - and the underlying electronic hardware is facing serious challenges ${ }^{3,4}$. Delivering the advances in electronics necessary to explore the full potential of the technology will require a workforce of scientists, engineers and technicians, with the necessary education to handle the very latest hardware.

In recent years, household electronics that inquisitive minds could disassemble and probe have all but disappeared, replaced by costly, complex and impenetrable devices. While these changes have, of course, led to enormous improvements in performance and capabilities, something has potentially been lost - an opportunity to try to understand. Learning the fundamental aspects of electronics is currently largely the reserve of courses beyond secondary education, with circuit and hardware design pushed to the latter years of higher education. Notably, devices such as the Raspberry Pi and Arduino have emerged, which provide a platform and community for teaching computer science and electronics to those with an interest. But a case for better electronics education at a rudimentary level remains.

An introduction to the basics of electronics was one of the key themes of the 49th edition of the International Physics Olympiad, which took place last month in Lisbon, Portugal (http://ipho2018.pt). The very first Physics Olympiad was held back in 1967 in Warsaw, Poland, and involved participants from only five countries.
This year, the event, which was organized by the Portuguese Physical Society, saw over 400 secondary school students from 87 countries come together to learn about and share their passion for physics - as well as to compete in experimental and theoretical competitions, each lasting five hours.

The students have no prior knowledge of the precise content of the competitions, and are given only a broad syllabus of theoretical, experimental and mathematical skills they should revise. Part of this year's experimental competition focused on paper transistors. In particular, the students were given a paper transistor and a junction fieldeffect transistor based on silicon, as well as the basic tools needed to characterize the devices (pictured). The aim of the exercise was to measure and plot the transfer and output curves for both devices, and extract their key characteristics such as their transconductance and pinch-off voltages. This challenging enough task was further complicated by the request to create and characterize a unipolar inverter using an additional load resistor.

The paper transistors used in the competition were those pioneered by the research groups of Elvira Fortunato and Rodrigo Martins, who are based at the Faculty of Science and Technology of the New University of Lisbon ${ }^{5}$. The devices use a cellulose (paper) membrane as both the dielectric and the substrate, and gallium indium zinc oxide as the semiconductor. They have potential applications in areas such as low-cost, smart packaging, and radiofrequency identification (RFID) tags for inventory management or food labels. For the competition, researchers from the groups of Fortunato and Martin fabricated and tested over 1,000 devices, which indicates something about the ease of fabricating these transistors. The devices also needed to survive transportation to the event, and a degree of rough handling during it, which further illustrates their potential robustness.

At the close of this year's International Physics Olympiad, $8 \%$ of the students received gold medals, $25 \%$ silver and

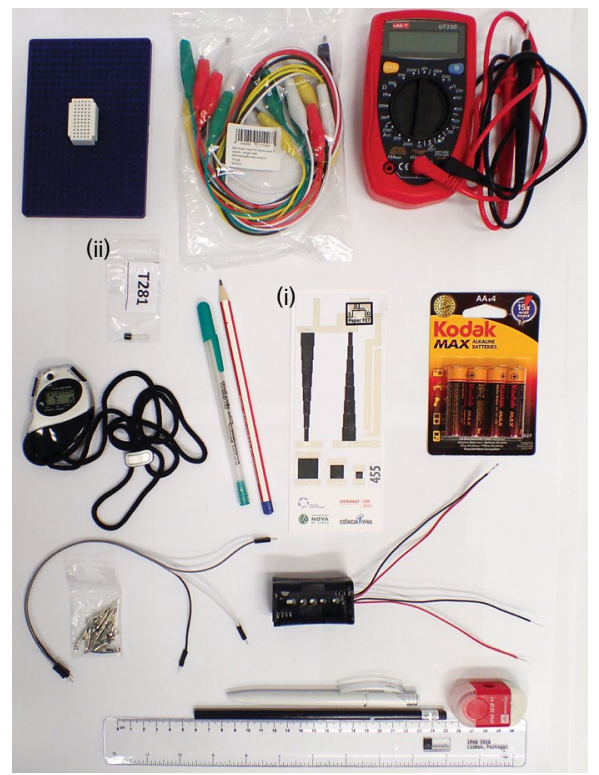

The selection of equipment and tools, which include a paper transistor (i) and a junction field-effect transistor (ii), supplied to students as part of the experimental competition of this year's International Physics Olympiad. Credit: José António Paixão

$50 \%$ bronze, and the student with the top score in both the experimental and theoretical exam was Yang Tianhua from China. All should go home proud of their achievements and, hopefully, inspired to learn more about the fundamentals and potential of electronics.

Published online: 13 August 2018 https://doi.org/10.1038/s41928-018-0128-7

\footnotetext{
References

1. After the reboot: computing education in UK schools. The Royal Society https://royalsociety.org/computing-education (10 November 2017)

2. Singer, N. How Silicon Valley pushed coding into American classrooms. The New York Times https://go.nature.com/2M6fol7 (27 June 2017).

3. Nat. Electron. 1, 205 (2018).

4. Salahuddin, S., Ni, K. \& Datta, S. Nat. Electron. https://doi. org/10.1038/s41928-018-0117-x (2018).

5. Fortunato, E. et al. IEEE Electron Device Lett. 29, 988-990 (2008).
} 